\title{
Novel Technique to Create a Secure Medical Image Watermarking
}

\author{
Priyanka \\ Department of Computer \\ Science and Engineering, \\ PG Scholar, SRMCEM, \\ Lucknow
}

\author{
Pankaj Kumar, PhD \\ Departmentl of Computer \\ Science and Engineering, \\ Assistant Professor, \\ SRMCEM, Lucknow
}

\author{
Raj Gaurang Tiwari, PhD \\ Department of Computer \\ Science and Engineering, \\ Assistant Professor, \\ SRMCEM, Lucknow
}

\begin{abstract}
The technique that hides the information within an original image without causing much affects on the originality of the image is known as digital image watermarking technique. This technique is mainly used for copyright protection or rightful ownership. The main objective of mostly all the techniques is to enhance the imperceptibility which is done by maintaining the robustness of the image. In this paper a new technique is proposed that is based on the frequency domain which is known as the SVD-DWT-DCT along with Kalman filtering. Comparisons are made in the earlier available as well as the new proposed technique. It is seen through the experimental results that the new proposed technique provides better results along the PSNR and MSE parameters.
\end{abstract}

\section{Keywords}

SVD, DWT, DCT, Kaman Filtering, PSNR, MSE

\section{INTRODUCTION}

The enhancement of achieved raw images from various sources such as cameras or sensors placed within the fields of various applications is known as image processing. The applications might involve the satellites, air ships etc as well as the images that are gathered from daily lives. Within the image processing, various techniques have been emerging recently, most of which include the techniques related to the enhancement of images gathered from the spacecrafts, military flights and so on [1]. There are various characteristics such as memory sizes of extensive sizes, graphics software, etc that have made the image processing technique gain popularity over other techniques. The classification method has been a difficult task to be executed for machines whereas an easy one for the humans [2]. There has been a growth in popularity of the object classification algorithms due to the high-capacity computers, the low-priced video cameras as well as the increasing demand of automatic video analysis. Within a simple classification system, the camera is fixed on a high interested zone and the processing of captured images is done further. Various steps that include image processing, object detection, object segmentation, feature extraction and object classification result in providing an efficient classification technique [3]. A database that has predefined patterns is present within the classification technique, from which the objects are compared and recognized. Through this, the objects are classified into appropriate categories.

The hiding of information behind an image such that there can be no manipulations made on its content is known as an image watermarking technique [4]. When it is seen that it is not possible for the intended node to extract the watermark content from the image, it is concluded that there are alterations made within the content of the respective image
[5]. There is only a single identity represented by the watermarking which is only known for the source node. The data can be hidden within the images. There is similar key to be used for inserting as well as extracting the data from the image. Within the least significant bit of an image, the data is hidden which is not to be seen though naked eye. It is almost impossible to commonly make a judgment if there is any data hidden or not. For the purpose of protecting data from fraud and forgery, the watermarking techniques have been used for authentication from earlier times [6]. The watermarking techniques are simpler as compared to other authenticating methods and are efficient in providing their purposes. The watermarks are applied to biometric data as well as identifiers through which the ownership is determined easily and it also helps in deterring their tamper. The Today, the usage of invisible watermarks is increasing as compared to the visible ones. The data within the invisible watermarks is not affected once it is embedded within it [7].

\section{LITERATURE REVIEW}

Nikita Kashyap, et.al proposed in this paper [8] an image watermarking technique that is based on the 3-level Discrete Wavelet Transform (DWT) technique. This technique is introduced for providing the basic copyright protection of data. With the help of the alpha blending method, a multi-bit watermark is embedded within the low frequency sub-band of a cover image. As compared to the other transform techniques, the insertion and extraction of the watermark in the grayscale cover image is easier. There are various parameters such as peak-signal-to-noise-ratio (PSNR) and mean square error (MSE) that are used for comparing the 1level and 2-level DWT based watermarking techniques. The results show that the new proposed technique provides better experimental results than other already existing techniques.

Chun-Shien $\mathrm{Lu}$ et.al proposed in this paper [9] a new multipurpose watermarking technique for copyright protection and content authentication. This technique involves the embedding of robust and fragile watermarks. There are two complementary watermarks embedded with the help of cocktail watermarking with the help of quantization of host image wavelet coefficients as MTUs (masking threshold units). Without the need of providing access to the host image, the embedded watermarks can be embedded very easily. There is a guarantee of at least one watermark to survive in a proper manner no matter which attack encounters. This ensures the protection of image. In terms of robustness and fragility, this method has provided efficient results which can be determined through the experimental results.

Navnidhi Chaturvedi, et.al proposed [10] that for the purpose of handling digital multimedia, the authenticity and copyright protection are the two distinct issues emerging with the 
technology advancement. For providing copyright protection, the image watermarking method is used with DWT. This method performs 2 Level Decomposition of original image and within Lowest Level (LL) sub band of the cover image, the watermark is embedded. For the purpose of achieving the original image back from a watermarked image, the Inverse DWT mechanism is utilized. The image is converted into blocks of $\mathrm{m}$ bits with the help of Discrete Cosine Transform (DCT). The reconstruction of the image is further done with the help of inverse DCT method. Various comparisons have been made amongst the DWT and the DCT-DWT techniques. The performance is compared on the basis of certain parameters and the results are compared.

Ali Al-Haj et.al [11] proposed a need of copyright enforcement technologies for the purpose of protecting the copyright ownership of various multimedia objects. There has been a rapid growth in the proliferation of digitized media with the increase of networked multimedia systems. An imperceptible and robust combined DWT-DCT digital image watermarking scheme is proposed within this paper. Through the combination of DWT and DCT, the provided digital image is watermarked. With the help of various experimental results achieved, it is seen that the performance of the watermarking algorithms is improved when the two transform techniques are combined as compared to the method that includes only DWT transform technique.

Surya Pratap Singh, et.al presented [12] a robust watermarking technique for color and grayscale image. For the purpose of providing secure and robust watermarking various methods are included within this new method. Within the 3 Level DWT, the watermark is embedded within this proposed technique. For providing security for image, the image is first passed through the chaotic encryption technique before embedding the watermark. In the form of DCT the watermark is embedded within this proposed method. A coefficient shifting algorithm is utilized for reducing the affects that are to be caused on the data of the main image. The technique has provided various robust results to the different image processing techniques for instance the JPEG compression, enhancement, resizing as well as the geometric operations.

\section{PROPOSED WORK}

Kalman Filter: There are various measurements that have been gathered over certain duration which have statistical noise and other issues. The utilization of these series of measurements for providing estimates of unknown variables is done with the help of Kalman Filtering technique which is also known as Linear Quadratic Estimation (LQE). The estimates that are made with the help of various collective measurements have proven to be more accurate as compared to the ones made with one measurement only. The Bayesian inference is utilized here and across the variables of each time frame, the joint probability distribution is estimated. There are two steps involved within this process. The first step is the prediction step in which the estimates related to the current state variables and their uncertainties are done with the help of Kalman filter. A weighted average is utilized for updating the estimates after the output of the next measurement is seen. Here, more weight is provided to the estimates that have higher certainty. This type of algorithm is a recursive algorithm which runs in real time with the help of present input measurements as well as the earlier calculated state and uncertainty matrix. There is no need for the additional past information here.
1. To study the various watermarking techniques through various mechanisms or schemes such as the Discrete Wavelet Transformation, Discrete Cosine Transformation and singular Value Decomposition.

2. To implement the DCT-SVD technique and analyze the processing time, computational power as well as robustness of the algorithm in order to prevent security attack.

3. To calculate the PNSR value of the DCT-SVD technique.

4. Applying Kalman filtering which increase the PSNR and increases the robustness of the algorithm which further results in enhancing the SVD, DCT and DWT technique.

5. The comparison of results of the earlier DCT-SVD technique and the enhanced DCT-SVD technique is to be done on the basis of the PSNR values. The graphic as well as tabular comparison of the robustness of these methods is done.

\section{EXPERIMENTAL RESULTS}

The proposed algorithm is executed with the help of MATLAB and the results achieved are analyzed in terms of MSE and PSNR values.

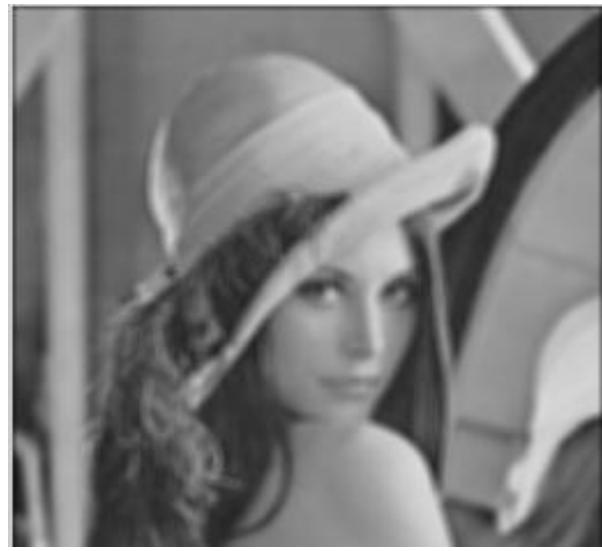

Fig 1: Watermarked Image

As shown in figure 1, the algorithm of DCT, DWT and SVD is applied which generate final watermarked image.

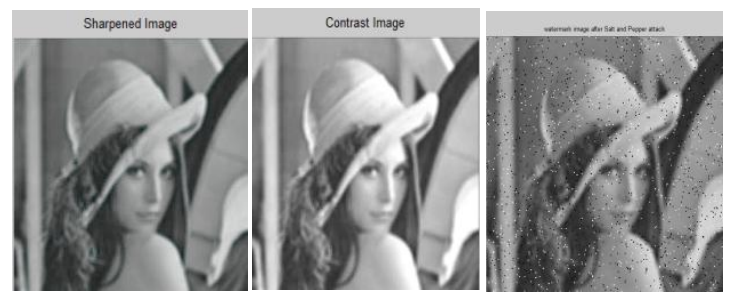

Fig 2: Security Analysis

As shown in figure 2, the reliability of the proposed system is tested under the various attacks which are shown in the figure. The attacks which test security of the system are Contrast attack, Sharpened and Salt \& Pepper attack. 


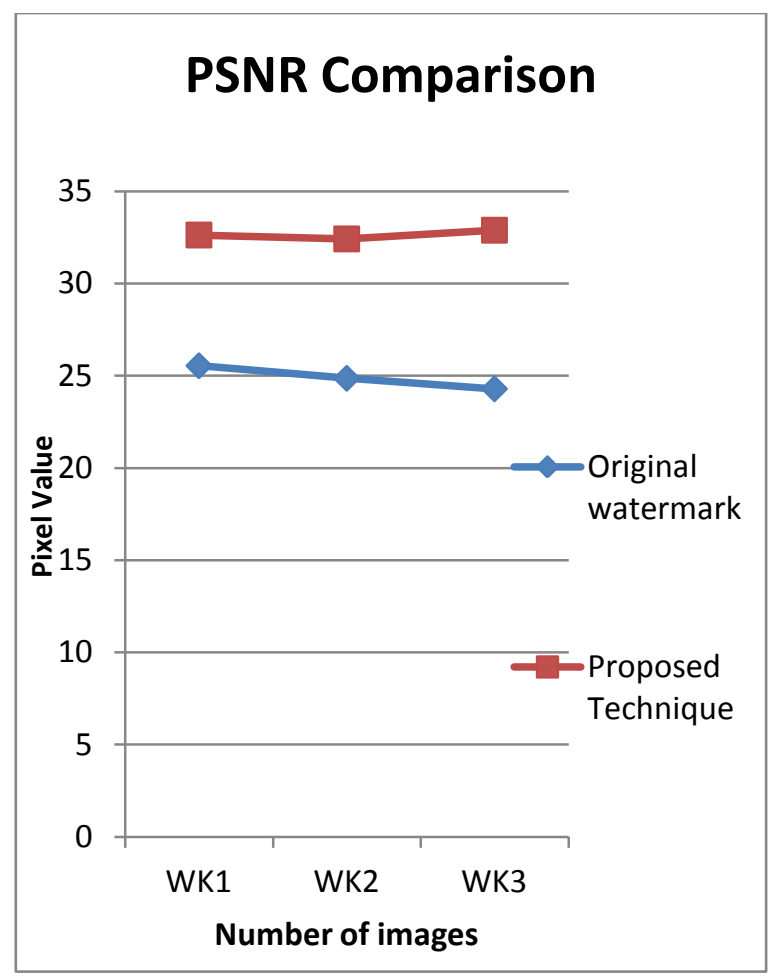

Fig. 3: PSNR comparison

As shown in the figure 3, the experimental results achieved show that the PSNR value of the proposed technique is more as compared to the PSNR of the original watermark technique.

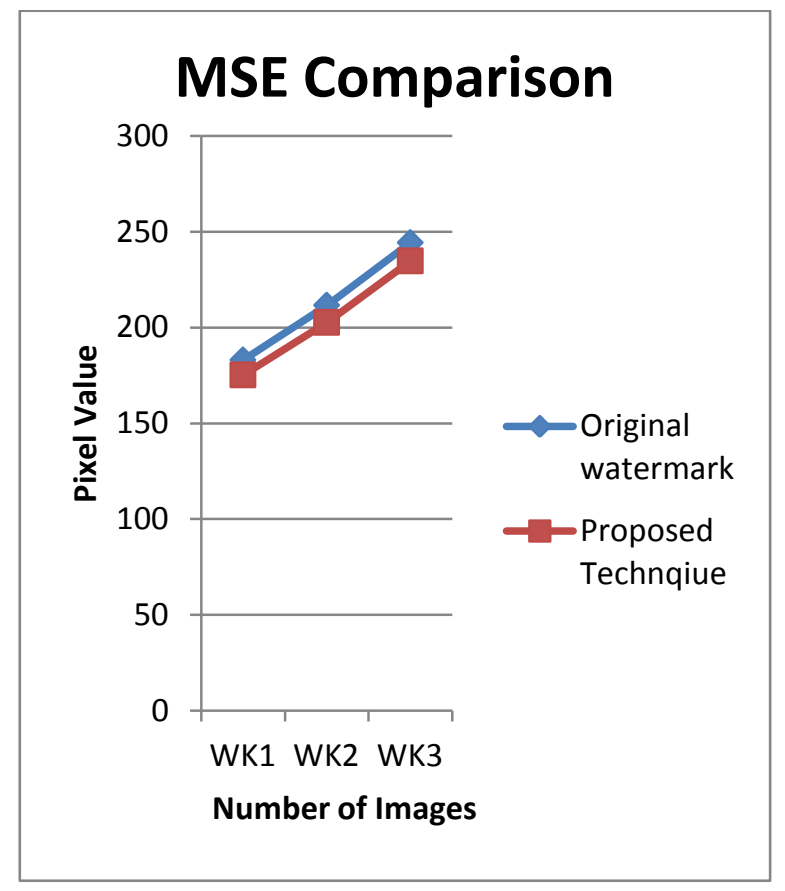

Fig. 4: MSE Comparison

As shown in the figure 4, it is seen by the experimental results achieved that the MSE value of the proposed method is less in comparison to that of the original watermarking technique.

\section{CONCLUSION}

There are certain objectives that are to be achieved within the digital image processing. In this paper, these objectives are met through the novel hybrid SVD-DWT-DCT technique. Two of the main objectives that are to be ensured within the watermark technique are robustness and imperceptibility of the image. As per these qualities the quality of a watermarked image can be determined. This also provides secrecy of the digital media. The acceptance values of the performance metrics are achieved which further help in providing an authorized digital image watermarking. The Kalman filter is used along with SVD-DCT-DWT technique and the PSNR and MSE values are achieved. The performance results of the new technique are better than the earlier technique. The achieved images are robust and can hide data from the authorized as well as unauthorized attacks.

\section{REFERENCES}

[1] Anthony T. S. Ho, Jun Shen, Soon Hie Tan, "A Robust Digital Image-in-Image Watermarking Algorithm Using the Fast Hadamard Transform", 2011, Springer

[2] Alexander Sverdlov, "Secure DCT-SVD Domain Image Watermarking: Embedding Data in All Frequencies", 2003, IEEE Region 10 Technical Conference on Convergent Technologies for the Asia-Pacific, Bangalore, India

[3] T. Serre, L. Wolf, S. Bileschi, M. Riesenhuber, and T. Poggio, "Object recognition with cortex-like mechanisms," 2007, IEEE Trans. on PAMI vol. 29, no. 3

[4] J.-K. Kamarainen, V. Kyrki, and H. K “ alvi“ ainen, "Invariance properties of Gabor filter based features overview and applications," 2006, IEEE Trans. on Image Processing, vol. 15, no. 5, pp. 1088-1099

[5] D. Gabor, "Theory of communication," 1946, Journal of Institution of Electrical Engineers, vol. 93, pp. 429-457

[6] Harshlata Vishwakarm, S. K. Katiyar, "Comparative Study Of Edge Detection Algorithms on the Remote Sensing Images Using MATLAB", 2012, International Journal of Advances in Engineering Research, Vol. No. 2, Issue No. VI, December ISSN: 2231-5152

[7] Priyanka, Rajesh Gulhane and V.T.Gaikwad, "International Journal of Advanced Research in Computer Science and Software Engineering", 2012, Volume 2, Issue 1, ISSN:2277 128X

[8] Kashyap, Nikita, and G. R. Sinha, "Image watermarking using 3-level discrete wavelet transform (DWT)", 2012, International Journal of Modern Education and Computer Science (IJMECS) 4.3: 50

[9] Lu, Chun-Shien, and H-YM Liao, "Multipurpose watermarking for image authentication and protection", 2001, Image Processing, IEEE Transactions on 10, no. 10: $1579-1592$

[10] Chaturvedi, Navnidhi, and S. J. Basha. "Comparison of Digital Image watermarking Methods DWT \& DWTDCT on the Basis of PSNR." image 2 (2012): 1

[11] Al-Haj, Ali, "Combined DWT-DCT digital image watermarking", 2007, Journal of computer science 3, no. 9: 740

[12] Singh, Surya Pratap, Paresh Rawat, and Sudhir Agrawal, "A robust watermarking approach using DCT-DWT", 
2012, International Journal of Emerging Technology and Advanced Engineering (ISSN 2250-2459, Volume 2, Issue 8

\section{AUTHOR PROFILE}

Priyanka received the B.tech. degree in Information Technology from Babu Banarasi Das Educational Society
Group of Institution Lucknow in 2014 and pursuing M.tech in Computer Science and Engineering from Shri Ramswaroop Memorial College of Engineering and Management, Lucknow during 2015-2017 and research on topic Security of medical image by watermarking using DWT-DCT-SVD under the guidance of Dr. Pankaj Kumar and Dr. Raj Gaurang Tiwari. 